\title{
Síndrome de Lesh-Nyham
}

Drs.(as): Sara Chernilo St., Federico Puga C.
Manuel Aspillaga H. e Isabcl Avendaño B. (1)

\author{
Lesh • Nyham Syndrome
}

Two cases of the Lesh-Nyham Syndrome are described. The metabolic origin of the disease is discussed, toghether with its sympoms, laboratory diagnosis and treatment with Alopurinol.

El síndrome de Lesh-Nyham.(1) descrito en 1964 es una enfermedad del metabolismo de las purinas que se transmite por un gen estructural ubicado en el cromosoma $X$, en forma recesiva $y$ de penetrancia completa.(2). Los niños afectados todos hombres, son aparentemente sanos hasta los 6 a 8 meses (3), y luego desarrollan el síndrome clínico caracterizado por: retardo mental, parálisis cerebral espástica, coreoatetosis, automutilación, hiperuricemia, litiasis urinaria, nefropat ia $y$ artritis gotosas y tofos. $(1,3,4,5)$. Estos enfermos presentan un déficit total de la enzima hipoxantina-guanina fosforil transferasa (HXGPT) $(3,4)$ lo que les ocasiona una incapacidad para sintetizar la hipoxantina y convertirla en Inosin Monofosfato (IMP) y Guanosín Monofosfato (GMP), a través de la vía endógena de síntesis de purinas. (6). Esta incapacidad los obliga a degradar toda la hipoxantina a ácido úrico, generando hiperuricemia e hiperuricosuria. Esto se asocia a acentuadas alteraciones en el metabolismo de las purinas: Elevación del índice de ac. úrico urinario y creatininuria, aumento en más o menos 20 veces de la excreción de ácido úrico después de la administración de glicina (14) y aumento del "turn over" del "pool" de ácido úrico en 2 ó 3 veces $(1,5)$. La HXGPT existe normalmente en todos los

(1) Médicos Pediatras Hospital Luis Calro Mackenna. tejidos del organismo (2), su concentración es mayor en los ganglios basales y en el cerebro (7) que en el resto de los tejidos.

Su presencia se puede detectar en cultivos de fibroblastos a los que se adiciona 6-mercaptopurina (8) o mediante electroforesis de proteína en los foliculos pilosos $(2,3)$.

En los niños afectados no se detecta la enzima en los glóbulos rojos (3) y las madres portadoras, clínicamente sanas, tienen un déficit parcial $(2,3)$.

Se han đescrito otras anomalías metabólicas de la sintesis de purinas: un déficit cualitativo de la HXGPT (9) y una alteración cuantitativa de la pirofosfato-ribosafosfato sintetasa $(11,12)$, ambas conducen en el niño a una enfermedad similar a la gota del adulto, pero que no se acompaña del cuadro neurológico característico del sínđrome de Lesh-Nyham, que seria exclusivo de aquellos casos que presentan un déficit total de la HXXGP descrito inicialmente por Lesh y Nyham. (1).

El pronóstico del cuadro es sombrio por cuanto la mayoría de los pacientes fallecen entre los $10 \mathrm{y}$ los 30 años (4), a pesar del tratamiento con Alopurinol que es capaz de mitigar y únicamente los sintomas secundarios de la hiperuricemia. (3). Las causas habituales de muerte son la insuficiencia renal aguda post renal (9), la insuficiencia renal crónica y la inanición (3).

Resulta interesante el hacer notar que este síndrome corresponde al primer déficit enzimático localizado, relacionado con una alteración conductual en el hombre: la automutilación. (2, 5, 12). 


\section{CASOS CLINICOS}

No 1 Marcel G.B. Fig. 1.
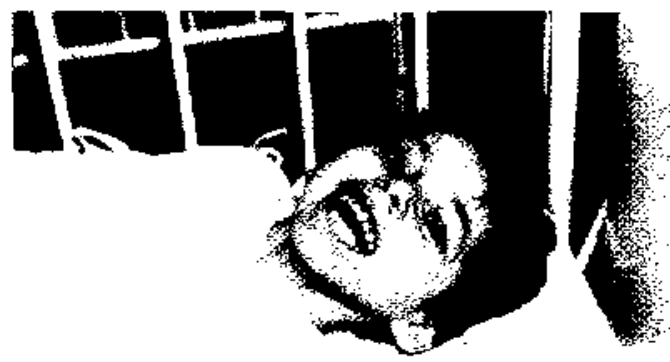

2

$$
\text { सुः }
$$

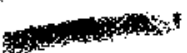

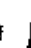

Figura 1

Niño de 4 años 9 meses enviado en Interconsulta al Hospital Luis Calvo Mackenna para estudio de un retardo mental severo con coreoatetosis y múltiples nutilaciones.

Antecedentes: Embarazo y parto normales. PN: $3860 \mathrm{gr}$. Al $5^{\circ}$ mes de vida la madre consulta por retraso en el desarrollo sicomotor diagnosticándose parálisis cerebral. Desde los 6 meses de edad, tiñe de rojo el pafral, el que además, ocasionalmente, contiene "arenillas". Ha sufrido 5 episodios de infección urinaria tratados con antibióticos, sin estudio radiológico. Poco antes del año comenzó a morderse el labio inferior y los dedos de las manos. No tiene antecedentes familiares de importancia.

Examen físico de ingreso: Peso $8 \mathrm{Kg}$. Estatura: $93 \mathrm{~cm}$., Circunferencia de Cráneo: $49 \mathrm{~cm}$.. Circunferencia del Tórax: $55 \mathrm{~cm}$. En malas condiciones generales, mal estado nutritivo, bajo de talla, inquieto, gritón, dice pocas palabras, obedece órdenes simples, se muerde constantemente labios y dedos, presenta por momentos intensos movimientos de extensión generalizados y además otros coreoatetósicos que se désencadenan ante cualquier estimulo. Hipertomía e hiperreflexia generalizadas de predominio derecho. Examen de Pares craneanos normal. Fig. 2.

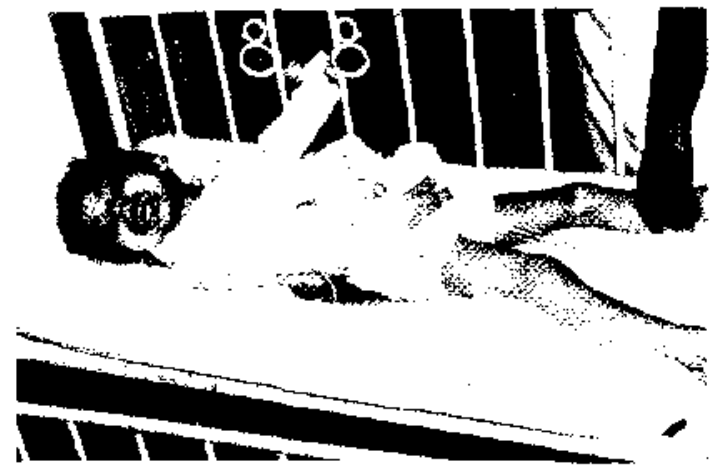

Figura 2
Exámenes de Laboratorio (Tabla 1):

Tabla 1 .

Exámenes de laboratorio

\begin{tabular}{|c|c|c|}
\hline & Caso No 1 & Caso $\mathrm{N}^{\circ} 2$ \\
\hline Orina & Sed. Normal & Sed. Normal \\
\hline Creatinemia & $0,4 \mathrm{mg} \%$ & $0,29 \mathrm{mg} \%$ \\
\hline Uricemia & $13,1 \mathrm{mg} \%$ & $9.9 \mathrm{mg} \%$ \\
\hline Ac. úrico orina & $108 \mathrm{mg} \%$ & $138 \mathrm{mg} \%$ \\
\hline Creatm. orina & $31 \mathrm{mg} \%$ & $23 \mathrm{mg} \%$ \\
\hline $\begin{array}{l}\text { Ac. úrico orina = } \\
\text { Creatín, orina }\end{array}$ & 3,5 & 6 \\
\hline Clearence de creatin. & $128 \mathrm{mg} \%$ & - \\
\hline Astrup y hemograma & Normales & Normales \\
\hline Transa. glut. piruv. & Normales & Normales \\
\hline Aminoacidemia & Normal & Normal \\
\hline Aminoaciduría & Levem. alter & Levemen. anorma \\
\hline Pielografía & - & $\begin{array}{l}\text { Dilat. pielouretera } \\
\text { bilateral. } \\
\text { Hidronef rosis. }\end{array}$ \\
\hline
\end{tabular}

Orina: pH 5, proteinas indicios, cilindros granuloso-céreos regular cantidad. Creatinemia: $0,4 \mathrm{mg} x$ dl. Uricemia: $13,1 \mathrm{mg} x \mathrm{dl}$. Ac. Urico en orina: $108 \mathrm{mg} x$ dl. Creatinina en orina: $31 \mathrm{mg}$ $\mathrm{x}$ dl.

Ac. úrico (orina) $=3,5$ Creatinina (orina). Clearence de creatinina $=128 \mathrm{mg} x \mathrm{dl}$. Astrup y hemograma normales. Transaminasa glutámico pirúvica $\mathrm{y}$ fosfatasas alcalinas normales. Amino. acidemia normal. Aminoaciduria levemente alte. rada.

Se inicia tratamiento con Alopurinol $50 \mathrm{mg} \mathrm{c} / 8$ horas y 5 días después, la uricemia ha descendido a $3,6 \mathrm{mg} \times \mathrm{dl}$. Se da de alta con indicación de tratamiento a permatiencia. (Tabla 2).

Tabla 2.

Niveles de uricemia antes y después de tratamiento con ajopurinol durante 3 días.

\begin{tabular}{lll}
\hline & Caso 1 & Casn 2 \\
\hline Nivis Inicial & $13,1 \mathrm{mg} \%$ & $9,9 \mathrm{mg} \%$ \\
Nivel Concrol & $3,6 \mathrm{mg} \%$ & $2,5 \mathrm{mg} \%$ \\
Dosis & $50 \mathrm{mg} \mathrm{c} / 8$ hirs. & $50 \mathrm{mg} \mathrm{ci8} \mathrm{hrs.}$ \\
\hline
\end{tabular}

No 2: Miguel G.B. Fig. 3.

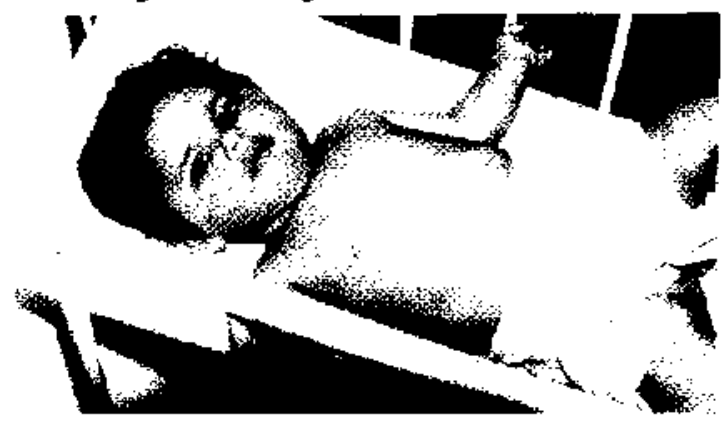

Figura 3 
Hermano del paciente anterior, consulta en Hospital Luis Calvo Mackenna a los 11 meses de edad. Parto y embarazo normales. PN $3550 \mathrm{gr}$. A los 3 meses no apoya la cabeza, a $\operatorname{los} 4$ meses sufrió un episodio de infección urinaria tratado con Gentamicina, sin estudio radiológico. Vuelve a consultar a los 6 meses diagnosticándose retraso psicomotor. A esa edad, llama la atención de los padres la coloración "zanahoria" de los pañales y la presencia de cristales 0 arenillas en los mismos.

Examen físico: Peso $9,4 \mathrm{Kg}$. Estatura: $74 \mathrm{~cm}$. Circunferencia de Cráneo: $44 \mathrm{~cm}$. Circunferencia de Tórax: 47,5 cm. En buenas condiciones de nutrición. Llora al movilizarlo, observa lo que lo rodea, responde a sonidos. No apoya la cabeza, no se sienta ni se pone de pie, no emite sonidos. Cambia objetos de una mano a otra con dificultad por la aparición de movimientos de extensión en los dedos, no lleva los objetos a la línea media. Hipertonía muscular generalizada en tubo de plomo, hiperreflexia osteotendinea generalizada con aumento del área reflexógena. Descargas tónicas frecuentes que comprometen las cuatro extremidades. Babinski $(+)$ bilateral. Pares craneanos normales.

\section{Exámenes de Laboratorio (Tabla 1):}

Orina: $\mathrm{pH} \mathrm{6}$, albúminas indicios. Cristales de urato abundantes. Creatinina: $0,29 \mathrm{mg} x \mathrm{dl}$. Uricemia $9,9 \mathrm{mg} \mathrm{x}$ dl. Ac. úrico (orina) $138 \mathrm{mg} \mathrm{x}$ di. Creatinina (orina) $23 \mathrm{mg} x \mathrm{dl}$.

Ac. Urico (orina) Creatinina (orina) $=6$. Astrup y Hemograma normales. Transaminasa glutámico pinivica y fosfatasas alcalinas normales. Aminoacidemia normal. Aminoaciduria generalizada, levemente anormal. Pielografía: dilatación pieloureteral bilateral, hidronefrosis. Se inicia tratamiento con Alopurinol $50 \mathrm{mg} \mathrm{c} / 8$ horas y 3 dias después se comprueba uricemia de $2,5 \mathrm{mg} \mathrm{x} \mathrm{dl}$. Se da de alta con indicaciones de mantener el trata. miento a permanencia. (Tabla 2).

\section{COMENTARIO}

la hiperuricemia es excepcional en el niño y su estudio es poco usual en la práctica pediátrica corriente.

Suelen tener hiperuricemia algunos casos de síndrome de Fanconi, la insuficiencia renal crónica, la enfermedad de Von Gierke tipo 1 (11), y la hipermielosis.

Sustancias tales como salicilatos, tiazidas, etambutol y plomo producen también aumento de la uricemia. En estos casos la hiperuricemia suele ser asintomática o excepcionalmente, presentarse como litiasis de la vía urinaria.
Ninguna de estas afecciones se acompaña del dan̉o neurológico aquí descrito, esto es: retardo mental, parálisis cerebral hipertonía, hiperreflexia, coreoatetosis y automutilación. Sin embargo, es frecuente encontrar en la práctica pediátrica muchos niffos con retardo mental y parálisis cerebral en los que no se ha hecho diagnóstico etiológico y valdria la pena realizar una uricemia con el objeto de identificar el problema cuando existe y dar consejo genético a los padres. $(2,3)$.

Falta efectuar en los dos casos presentados, mediciones de actividad de HXGPT para certificar el diagnóstico, sin embargo no dudamos de éste porque el cuadro clínico de nuestros enfermos corresponde exactamente con lo descrito por Lesh-Nyham y otros $(1,3,4,5)$. Como además ambes son de sexo masculino, hijos de padres sanos, es altamente probable la transmisión por un gen recesivo, ligado al sexo y de penetrancia completa.

\section{BIBLIOGR AFIA}

${ }^{1}$ Lesh M., W.L. Nyhom: A familial Disouder of Uric Acid Metabolism and Central Nervous System Function. Am. J.M. 36: 561, 1964.

2 V. Franke, J, Felsenstein, S.M. Gartier, B.R. Miglon, J. Doncis, J, Esecgmiller, F. Bakay, W.L. Nyham.: Tha Occurence of New Mutants in the X.-Linked Recessive Lesh-Nyhan Discase. Am. J. Hun. Genet. 28 (2) 123 March 1976.

3 W.L. Nyham. The Lesh-Nyham,: Syntrome, Annu Rev. Med. 24: 41, 1973.

4 w.L. Nyham, W.J. Oliver, M. Lesh.: A Familial Disorder Of. Uric Acid Metabolism and Central Nervous System Function 11. J. Pediatric 67: 253, 1965.

s W.J. Nyham.: A. Disorder of. Uric Acid Metabolism and Cerabral Function in Childhood. Arthritis Reum 8: $659,1965$.

${ }^{6} N$. Lottrence Edward, D. Recker, I.H. Fox.: Over Production of Uric Acid in Fipoxanthine - Guanine Phosphoribosiltransficase Deficiency. J. Clin. Invest. 63: (S) 922, May 1979.

7 A.C. Allison, T. Ilovi. R. W.E. Wotts, A.D. B. Webster.: Inmulugical Observations an Patients With Lesh. Nyham Syndrome and tha Role of de - Novo Purine Synthesis in Lymphocite Transtomation. Lancet: 2 (7946) 1179,13 Dic. 1975.

${ }^{8}$ J.E. Seegmiller, F.M. Rosembloom. W. Kelly.: Tnzime Defect Associaced with a Sex Linked Human Neurologucaf Disorder and lixcesive Purine Syntheris. Science 155: 1682, 1967.

9 L. Sweetman. M.A. Hoch, B. Bakay, M. Borden, P. Lesh, W.L. Nyham.: A Disctint Human Variant of Hipoxanthinegualine Phosphoribosyl Transferase. J. Pediat. 92 (3) 385, March 1978.

10 J.B. Wyngaarden.: Gout and other disorders of UJic Acid Metabolism, Hatrison's ]'rinciples of Internal Medicine. Mc. Graw Hill Book Company. Sew York, 1974, Seventh Ed.

I I Bauzá, R. Hernández, F. Monckeber.: Enfermedades Genéticas y Citogenéticas en el nir̉o. Ed. Universitaria. Santiago, 1973. 